\title{
Masculin et féminin dans la musique de la Rome antique
}

De la théorie musicale à la pratique instrumentale

\section{Christophe VENDRIES}

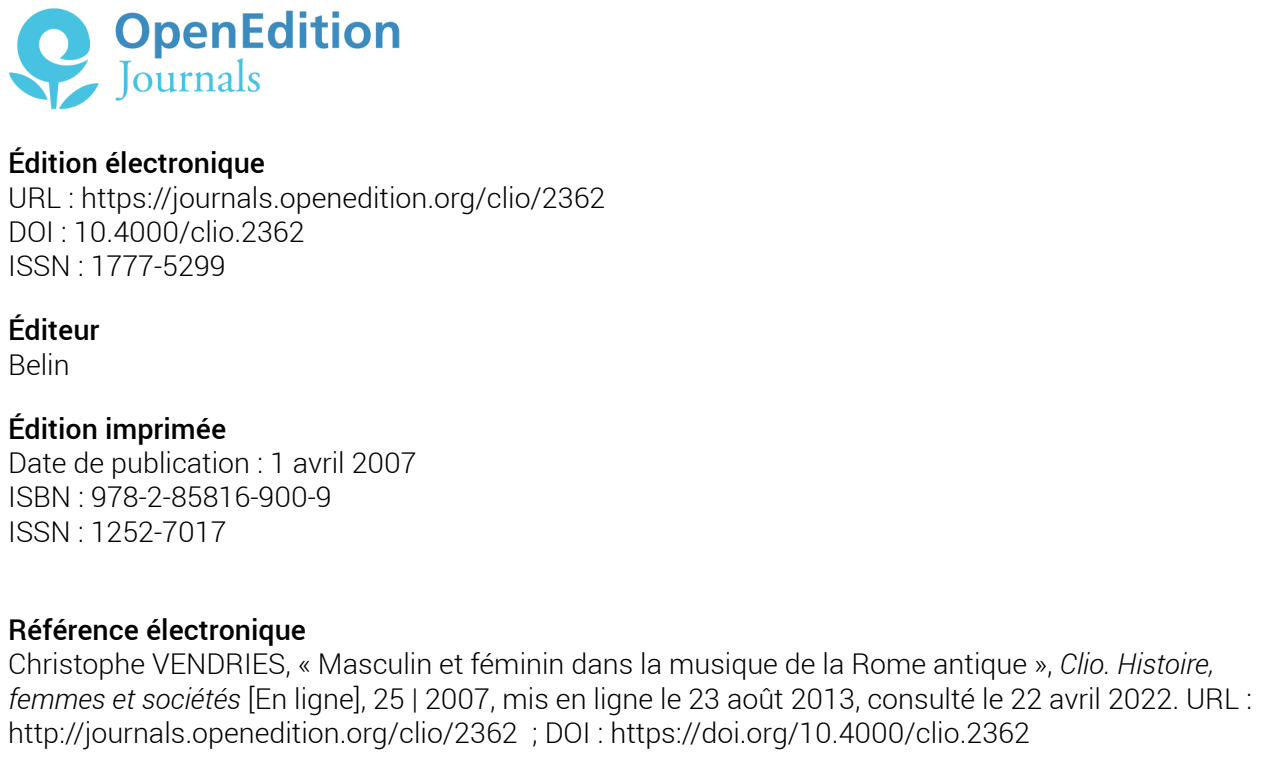

Ce document a été généré automatiquement le 22 avril 2022.

Tous droits réservés 


\section{Masculin et féminin dans la musique de la Rome antique}

De la théorie musicale à la pratique instrumentale

\section{Christophe VENDRIES}

1 Le lien entre genre et musique n'est pas forcément aisé à concevoir, à formuler et à illustrer à partir de l'exemple romain. Les recherches sur la musique antique demeurent peu avancées dans le domaine de l'imaginaire et des représentations; aussi les questions du partage entre les sexes, de la sexualisation des instruments de musique - parfois posées pour d'autres périodes ou d'autres disciplines ${ }^{1}$ - et du rapport entre genre et pratique musicale restent en grande partie à écrire. Néanmoins, l'approche de la musique ne peut-elle être l'occasion de valider certains apports méthodologiques et conceptuels de la notion de genre à travers une lecture sexuée de cet art? En d'autres termes, peut-on, à partir de l'exemple de la musique aborder la question du rapport entre les sexes?

2 Nos sources laissent deviner que la problématique du genre se manifeste de plusieurs façons et à plusieurs niveaux : non seulement, comme on pouvait s'y attendre, à travers les pratiques musicales sous la forme de prescriptions ou d'interdits, mais aussi grâce aux éléments de la théorie musicale (sons, "modes", rythme et mélodie). Il faut prendre en compte également la classification des instruments de musique. Dans le domaine musical comme en d'autres, l'enquête montre que la frontière entre les sexes est parfois mouvante.

\section{Les leçons de la théorie musicale : le partage entre le masculin et le féminin}

3 Plusieurs auteurs de l'époque impériale ont laissé des traités de théorie musicale rédigés en grec puisque la Grèce était la référence absolue en matière de musique. Ces textes donnent une idée assez précise de ce qu'était la conception de la musique chez les Anciens, du moins sous la forme d'une réflexion intellectuelle et philosophique. 
4 La lecture du traité musical du théoricien Aristide Quintilien, qui vivait probablement au début de l'Empire, est éclairante sur bien des points ${ }^{2}$ car cet ouvrage peut être considéré comme une synthèse des connaissances sur la musique gréco-romaine. Il dépasse de loin la stricte acception musicale et aborde des notions qui touchent autant à la philosophie, à l'ethos et aux rapports entre la musique et les réalités de la vie humaine. Le discours sexué est bien présent tout au long de ce traité car le théoricien annonce en préambule du second chapitre (consacré à l'éducation par la musique) que «le rythme est à l'harmonie (harmonia) comme le masculin au féminin (arrènos pros thèlu)» en reprenant là une classification courante chez les Anciens concernant le rythme mâle et la mélodie féminine.

5 Selon le théoricien, il existe des sons masculins et féminins (II, 12) : «certains sont fermes (stereoi) et masculins, d'autres sont plus relâchés (aneimenoi) et plus féminins »; il est aussi des sons mixtes (heteroi metaxu) « qui participent des deux caractères ». La distinction entre les deux passe par l'utilisation de certaines voyelles dans le système de solmisation: pour solfier un air simple, les Grecs et les Romains utilisaient des voyelles dites féminines ou masculines (II, 13). Celles qui ouvrent grandement la bouche ont « des sonorités majestueuses et viriles " alors que celles « qui étirent la bouche en largeur ont une prononciation plus faible et plus féminine »; par exemple, l'oméga est masculin "puisqu'arrondi et dense» alors que l'êta est féminin: "quand on le prononce, le souffle se dissipe en quelque sorte et se trouve filtré». Et d'ajouter plus loin : «les sons (phtongoi) qu'on énonce avec êta sont mous et efféminés (pathètikoi kai tethèlusmenoi); ceux qu'on énonce avec oméga sont actifs et virils (drastèrioi kai errenomenoi) » (II, 14). Cette distinction, comme l'explique F. Duysinx ${ }^{3}$, semble venir du fait que les Ioniens, qui passaient pour efféminés dans la tradition, utilisaient l'êta là où, dans les dialectes doriens, on employait l'alpha.

6 Aristide Quintilien passe également en revue ce qu'il est convenu d'appeler par habitude les « modes » grecs 4 (II, 14) - en réalité les tropes - qui se prêtent tout autant à une division entre masculin et féminin : parmi les tons $(\text { tropoi })^{5}$, le dorien est considéré comme le plus grave (barutatos) et « convient au caractère mâle » tandis que la tonalité phrygienne est plus féminine. Comme pour les sons, le reste des «modes » (l'iastien, l'éolien) est regardé comme "intermédiaire ». Fidèle à la tradition antique, Aristide Quintilien martèle que la supériorité naturelle revient au mâle dans la musique et que ce qui relève du féminin est par nature imparfait.

7 Ce discours sur les sons et les modes débouche tout naturellement sur l'emploi circonstancié des instruments de musique. Non content d'introduire la connotation sexuée des sons et des tons, Aristide Quintilien aboutit logiquement à la division des instruments de musique (II, 14) :

« ceux [les instruments de musique] qui descendent du proslambanomène [son le plus grave du système] du ton (tropos) dorien sont les plus caverneux et possèdent un caractère mâle; ceux qui montent à l'aigu jusqu'au diatonos sont de caractère mixte ; ceux enfin qui viennent après ceux là, en montant davantage, sont les plus aigus et les plus féminins ".

8 Il livre le détail de sa pensée (II, 16) : dans les instruments à vent, la trompette (salpinx) a forcément un caractère mâle " en raison de sa puissance » alors que l'aulos ${ }^{6}$ phrygien, "plaintif et funèbre » est connoté féminin - ce qui, a priori, ne laisse pas d'intriguer quand on sait que sa sonorité était grave ${ }^{7}$-. Cela s'explique par le fait que les Phrygiens, comme les autres peuples d'Asie Mineure, passaient pour être efféminés . Quant aux autres auloi (aulos pythikos et aulos chorikos ${ }^{8}$ ), ils sont présentés comme intermédiaires 
(mesoi). Parmi les instruments à cordes, la lyre correspond au masculin tandis que la harpe (la sambykè) est liée au monde féminin parce qu'avec "son extrême acuité (oxuthetos), due à la petitesse de ses cordes, elle mène au relâchement (eklusin periagousan)». Quant à la cithara, elle relève des catégories dites intermédiaires. Quintilien ne dit pas autre chose dans son traité oratoire: il répète que la lyre correspond au caractère mâle et la harpe (sambuca) au caractère féminin (Institution oratoire, I, 101).

Les instruments de musique ont-ils un sexe ? A en croire les théoriciens, il se pourrait bien que oui. Lorsqu'ils traitent de la nomenclature instrumentale dans leur discours, ils ont coutume de distinguer ce qui relève du féminin et du masculin. Aristoxène de Tarente, le premier théoricien grec de la musique au $\mathrm{IV}^{\mathrm{e}} \mathrm{s}$. av. J.-C., avait déjà pris l'habitude de classer parmi les plus aigus les auloi virginaux (parthenoi) et juvéniles (paidikoi), c'est-à-dire avec une tessiture de jeune fille et de jeune garçon. Dans certaines sociétés, c'est la forme de l'instrument qui permet de le rattacher à la sphère du masculin ou du féminin (comme le tambour à fente aux Nouvelles Hébrides); dans la pensée romaine, si l'anthropomorphisme des instruments de musique est mis en avant par les philosophes, c'est d'abord la tessiture ${ }^{9}$ qui fait entrer l'instrument dans la catégorie masculine ou féminine.

On ne saurait limiter pour autant l'approche du genre dans le champ musical au seul discours théorique car bien des usages propres au monde des musiciens permettent de soulever la question de leur appartenance à la sphère du masculin ou du féminin.

\section{L'ambiguïté du citharède à Rome : efféminé, infibulé... mais séducteur de femmes}

11 A l'instar des acteurs connotés par la tradition comme des gens efféminés ${ }^{10}$, certains musiciens, et à leur tête les citharôdes (les chanteurs s'accompagnant à la cithara), ont la réputation d'être des personnages ambivalents. Leur allure concourt à introduire une sorte d'ambiguïté: ils sont glabres, portent souvent une chevelure longue comme Apollon, et surtout vêtus d'une grande robe ample avec une ceinture identique à celle que portent les Muses et que les Romains appellent palla, syrma ou stola (FIG. 1). Apulée décrivant la statue d'un citharède insiste sur «sa tunique aux broderies multicolores [qui] tombe jusque sur ses pieds, retenue par une ceinture à la grecque » (Florides, XV, 8). Les stéréotypes sont bien ancrés : ce n'est pas un hasard si, dans le discours que Dion Cassius (LXII, 6, 3) prête à la reine barbare Boudicca dans l'île de Bretagne, il lui fait déclarer à propos de l'empereur Néron : « c'est une femme (gunè) et la preuve c'est qu'il chante, joue de la cithara et s'occupe à se parer (aidei kai kitharizei kai kallôpizetai) ». Et le philosophe Sénèque de s'interroger: "n'est-ce pas vivre contre la nature que de prendre des vêtements féminins?»(Lettres à Lucillius, 122,7) ${ }^{11}$. C'est pourquoi les musiciens, les acteurs et, pire encore, les danseurs - les deux derniers se confondent dans l'Antiquité - sont souvent suspectés de mollitia. Libanios invective les danseurs de pantomime en des termes peu amènes: «leurs longs cheveux, leurs vêtements de femmes riches et brodés d'or témoignent de leur luxure » (Discours, LXIV, 31-34) ${ }^{12}$. C'est d'autant plus vrai que la fonction de danseur à Rome est assumée essentiellement par des hommes puisque les femmes sont quasiment absentes de la scène des théâtres ${ }^{13}$. Le danseur, l'acteur et le musicien se voient pêle-mêle appliquer d'autorité la définition normative de la féminité en vertu des critères de l'apparence. 
12 Le paradoxe de tout cela tient au fait que les citharèdes, dépeints communément comme des êtres efféminés, plaisent en réalité beaucoup aux femmes, y compris lorsqu'ils portent une fibule sur le sexe (un anneau de métal fixé à l'extrémité du gland) qui leur interdit toute forme de rapport sexuel ${ }^{14}$. Cette opération appelée infibulation était préconisée pour les tragédiens et les citharèdes « dans l'intérêt de leur voix » nous dit le médecin Celse ; elle participe de cette volonté de réguler la sexualité des gens de spectacle - sportifs de haut niveau et chanteurs - au motif que les rapports amoureux passaient pour nuire à la santé. Elle n'a rien à voir avec la castration qui était connue dans la Rome antique, mais qui ne fut jamais appliquée dans un but musical comme on le fit à Naples à l'époque baroque. Il est étonnant de voir apparaître l'adjectif « vierge » en conclusion de l'épitaphe d'un citharède retrouvée à Rome ${ }^{15}$ comme s'il s'agissait d'un titre de gloire. Un proverbe latin disait d'ailleurs qu'on ne pouvait être: « plus abstinent qu'un citharède ». Mais il ne faudrait pas croire pour autant que l'infibulation était généralisée: même le médecin Celse reconnaissait que cette opération était souvent plus « superflue que nécessaire » et cela semblait davantage relever d'une sorte d'idéal que les satiristes de l'époque impériale se plaisaient à tourner en ridicule. D'où la formule sarcastique de Juvénal (VI, 379) à propos d'une matrone romaine : « Aime-telle le chant? Nul ne gardera sa fibule intacte parmi ceux qui vendent leur voix aux préteurs ", faisant allusion aux célèbres citharèdes qui louaient leur talent aux magistrats organisateurs des jeux. Ainsi, bien que dépeints comme efféminés, les citharèdes sont aussi montrés comme des hommes qui oscillent entre la contrainte de la fibule - gage présumé de la préservation de leur voix et symbole de continence amoureuse - et le désir de plaire aux femmes et de les satisfaire sexuellement.

\section{L'histoire du travestissement de Clodius en psaltria}

13 L'ambivalence homme/femme chez les artistes passe forcément par le thème du travestissement, qui est au cœur des études sur le genre ${ }^{16}$ et qui invite à poser sans détours la question de la définition des sexes. Cette idée prend corps chez les musiciens à travers deux célèbres histoires : celle, réelle, de Clodius lors des rites du culte de Bona Dea à Rome au dernier siècle de la République et celle, mythique, d'Achille, caché à la cour du roi Lykomède qui trouve une expression iconographique très aboutie à l'époque impériale.

14 Ce n'est pas un hasard si l'histoire rocambolesque de Clodius, qui se déroule en 62 av. J.C. dans le sanctuaire de Bona Dea, fut choisie comme exemple par John Scheid pour illustrer un passage de l'Histoire des femmes concernant le féminin dans le rituel ${ }^{17}$ : on trouve là le cas d'un culte public célébré exclusivement par les matrones. L'histoire est celle de la transgression d'un espace réservé aux femmes, en l'occurrence la domus publica de César grand pontife, ce qui en fait un délit religieux. Un jeune patricien, Publius Clodius Pulcher, s'y introduit nuitamment grâce à la complicité d'une esclave afin de séduire l'épouse de César, Pompéia, occupée à célébrer une cérémonie secrète lors de la fête annuelle de la Bona Dea qui réunissait les Vestales et les dames de l'aristocratie romaine ${ }^{18}$. Clodius se déguisa en femme (muliebri vestitu nous dit Cicéron) et plus précisément en joueuse d'instruments à cordes car le sacrifice était accompagné de chants et de danses. Cette musicienne est qualifiée par Cicéron, Juvénal ou Plutarque de psaltria, terme qui peut désigner autant la cithariste que la harpiste ${ }^{19}$ parce que psallein en grec veut dire «jouer avec les deux mains ». Il est délicat de trancher mais, 
étant donnée l'accumulation des attributs féminins dans les descriptions de Clodius, la harpe conviendrait mieux parce qu'elle est connotée dans l'imaginaire collectif des Romains comme un attribut féminin - bien plus que ne l'est la cithara - et oriental. C'est d'autant plus vrai que Cicéron, pour vilipender Clodius, insiste à la fois sur les références au féminin et sur les allusions à l'Orient en introduisant dans son discours un certain nombre de mots d'origine étrangère ${ }^{20}$ :

«P. Clodius a quitté une robe de safran (crocota), un turban (mitra), des sandales de femme, des bandelettes de pourpre, un soutien-gorge (strophio), une harpe (psalterium), la turpitude, le scandale, pour devenir soudain ami du peuple» (Sur la réponse des haruspices, 44$)$.

15 Finalement, l'intrus fut démasqué, trahi par sa voix mâle comme le raconte Plutarque (Cicéron, 28, 3), ce qui provoqua un scandale retentissant. Ce déguisement valut à Clodius les sarcasmes de Cicéron qui put dès lors mettre en doute la virilité de son adversaire lors du procès car à Rome, comme on l'a vu, le vêtement est très codifiéé ${ }^{21}$ et le travestissement en femme s'accompagne aussitôt de l'accusation d'effémination et d'impudicitia. La dialectique utilisée par Cicéron est sans équivoque :

«Monstre! tu n'as pas honte de souiller ce temple, cette ville, cet air, ce jour? Toi qui as revêtu les habits d'une femme, tu oses faire entendre une voix d'homme (virilem vocem) (...) » (Cicéron, P. Clodium et Curionem, V).

Tout cela est contraire aux valeurs qui structurent l'homme libre à Rome, et en particulier à la dignitas. Quelles conclusions en tirer? "D'une manière générale, il apparaît que l'identification des genres exige, à Rome comme dans la plupart des autres civilisations, le respect de la culture des apparences sexuées ${ }^{22}$. Et surtout, cette affaire Clodius démontre « que le genre n'est pas le sexe $»^{23}$.

\section{La leçon du mythe d'Achille à Skyros : de la féminité de la cithara à la virilité de la trompette}

La fable, que l'on trouve chez les poètes postérieurs à Homère ${ }^{24}$, est celle de la fuite d'Achille dans l'île de Skyros. Un destin tragique lui avait été prédit s'il partait combattre car un oracle avait révélé qu'il devait mourir devant Troie : Pélée, son père, (ou Thétis, sa mère, selon les versions) imagina de cacher Achille en le déguisant en jeune fille dans le gynécée du roi Lykomède. Le fils de Thétis adopta alors une longue chevelure (on l'aurait appelé Pyrrha, "la rousse»), se para de bijoux, et serait resté neuf ans à Skyros où il passa son temps à jouer de la cithara dont le centaure Chiron lui avait autrefois enseigné les rudiments. Voici ce qu'en dit Ovide qui imagine le dialogue entre Achille et Briséis, son esclave :

«Les Grecs te croient désolé ; cependant tu bouges le plectre ; contre son sein tiède, une tendre amie te retient, et si quelqu'un demande pourquoi tu refuses de combattre: " le combat est malfaisant; la cithara, le chant et l'amour sont agréables. Il est plus sûr d'être étendu sur un lit, après avoir étreint une femme, de pincer de ses doigts une lyre de Thrace, que de soutenir de ses mains le bouclier et la lance à pointe aiguë, et sur sa chevelure la pression du casque » (Héroïdes, III, 113-120 ; trad. M. Prévost, CUF, modifiée).

18 Achille devient le parangon du guerrier renonçant volontairement, mais momentanément, à sa virilité. Cet épisode de la cachette d'Achille est décrit par Stace qui composa à la fin $\mathrm{du} \mathrm{I}^{\mathrm{er}} \mathrm{s}$. une épopée dédiée à Domitien dans laquelle il se proposa de narrer toute la vie du héros. Elle resta finalement inachevée, mais il est le seul dans 
la littérature à raconter les leçons de musique données par Achille à Déidamie, la fille du roi, sans que l'on sache à quelle source il a pu puiser (Achilléide, I, 574-576). L'histoire n'en prend que plus de relief quand on sait que la femme de Stace, Claudia, était musicienne. Certains se sont demandés si Stace ne s'était pas inspiré de l'affaire Clodius pour raconter l'histoire d'Achille caché parmi les filles de la cour du roi Lykomède car on découvre un passage qui semble y faire allusion :

« une redoutable prêtresse se tient à la limite prescrite et scrute les abords afin qu'aucun profanateur ne vienne se glisser dans le cortège des femmes (Achilléide, I, 600-603, trad. J. Meheust, CUF). ${ }^{25}$.

19 Finalement Ulysse apprit que Troie ne pourrait être prise sans Achille; alors, après avoir découvert l'endroit où il se cachait, il imagina d'envoyer un trompette afin de réveiller en lui l'instinct guerrier.

«Ces présents, lui dit-il, pour peu qu'il se cache par ruse au milieu des filles de la cour de Lycomède arracheront au fils de Pélée un aveu spontané et l'amèneront aux combats. Pour toi souviens toi, lorsque ce sera le moment, de tout apporter rapidement du vaisseau et de joindre à ces présents un superbe bouclier ouvragé et ciselé d'or ; ma lance suffira ; qu'Agyrte le bon trompette, soit là avec toi, et qu'il cache son instrument en l'apportant, car c'est pour un usage secret » (I, 719-725).

A l'appel de la trompe, Achille réclama aussitôt des armes :

« Il enlevait déjà sa robe quand, selon les ordres reçus, Agyrte donna un grand coup de trompette (cum grande tuba sic iussus Agyrtes insonuit) : c'est la débandade chez les jeunes filles, qui jettent les présents de tous côtés, vont pleurer près de leur père et croient le combat engagé. Mais, sans qu'il y eût touché, les vêtements sont tombés de la poitrine du héros, déjà le bouclier et la lance trop courte, disparaissent au creux de sa main » (I, 874-879).

21 Sans doute la sonnerie jouée par Agyrte était-elle celle de l'avertissement (exormètikon) qui, selon Pollux (Onomasticon, IV, 86), servait à donner le signal du réveil ou du départ. Cette intervention de la trompette n'est mentionnée que dans les sources tardives (Apollodore, Hygin, Stace et Philostrate le Jeune), mais ce récit existait déjà peut-être dans les Skyroioi d'Euripide ${ }^{26}$. Cet épisode, que les modernes appellent «la scène de la révélation $»^{27}$, eut un large écho dans l'iconographie impériale alors qu'il n'avait pas connu de réel développement littéraire. On le trouve dès le $\mathrm{I}^{\mathrm{er}}$ siècle dans la peinture murale (Pompéi, VI, 15, 1) ${ }^{28}$, mais cette éclosion est surtout perceptible à une époque tardive ${ }^{29}$. En effet, ce thème rencontra un succès certain sur les sarcophages à reliefs, et en particulier ceux qui étaient fabriqués dans les ateliers athéniens aux $\mathrm{II}^{\mathrm{e}}-\mathrm{III}^{\mathrm{e}} \mathrm{s}$. Il faut aussi prendre en compte les pavements de mosaïque qui ornaient parfois des salles destinées aux femmes ${ }^{30}$, le décor de l'argenterie. ${ }^{31}$ et bien d'autres supports. Pour autant l'influence directe de Stace sur les sculpteurs et autres artistes n'est pas attestée. Il est probable que les reliefs se rattachent à une tradition plus ancienne, dont Stace s'est peut-être lui même inspiréés2.

Le séjour d'Achille auprès des filles de Lykomède est donc l'un des sujets les plus souvent traités dans l'art funéraire des sarcophages romains ${ }^{33}$. Achille est montré dans le gynécée jouant de la cithara ou de la lyre, habillé en femme, seul ou entouré de Déidamie la fille du roi et de jeunes femmes qui vaquent à des occupations éminemment féminines : elles filent la laine ${ }^{34}$ ou jouent de la musique. Pour mettre en scène cette histoire, les sculpteurs ont retenu plusieurs motifs ${ }^{35}$. On trouve souvent sur un petit côté du sarcophage un raccourci des deux épisodes comme c'est le cas sur un exemplaire conservé au Musée du Louvre ${ }^{36}$ : Achille assis joue de la cithara entouré par les filles du roi (Déidamie est installée sous l'arcade tandis que sa sœur tient également 
un instrument à cordes) ; à l'extrémité une vieille esclave a disposé à ses pieds un pot rempli de laine - symbole éminemment féminin des vertus domestiques - tandis qu'à l'arrière-plan un guerrier casqué souffle dans une longue trompette droite (FIG. 4). Lorsqu'il entend l'appel de la trompe guerrière, Achille, « qui dissimulait son sexe sous la longue robe des femmes » comme le raconte si bien Ovide, abandonne ces attributs féminins et reprend les armes pour rejoindre le champ de bataille. Alors qu'est posée la question de l'identité du héros, on devine que la leçon est claire : la mollitia laisse place à la virtus retrouvée.

Cette histoire, qui illustre le jeu sur le féminin et le masculin, a nourri dans la littérature latine nombre d'allusions grivoises à propos de la lyre d'Achille. Varron par exemple utilise une phrase à double sens lorsqu'il écrit : «ils lui demandent de faire sortir sa Briséis [son esclave] qui maniait son instrument (nervia tractare)» (Onos lyras, XIX, 366) ${ }^{37}$. C'est le cas également d'une épigramme tirée des Priapea qui se propose de revisiter cette fable : «il bandait plus dur que les cordes de sa cithara» (ad citharam cithara tensior ipse sua ${ }^{38}$ ) écrit-on en faisant allusion à l'épreuve de l'arc organisée par Pénélope lors du retour d'Ulysse à Ithaque. On retrouve à nouveau ce paradoxe entre la féminité apparente du personnage et sa capacité à séduire les femmes par la musique de sa lyre qui devient ici l'instrument d'un jeu érotique.

Il y a lieu de s'interroger sur les raisons du succès iconographique de cet épisode. Bien entendu, c'est l'opposition entre le jeu de l'instrument à cordes pensé comme féminin et la trompette liée au monde guerrier qui est ici mise en exergue. Selon F. Cumont, la série des sarcophages d'Achille à Skyros exaltait indirectement la virtus de tous ceux qui, comme le héros, avaient préféré aux loisirs efféminés la gloire de vivre dangereusement ${ }^{39}$. L'exemple d'Achille servit à justifier la pratique des instruments à cordes chez certains nobles comme Pison, gagné par la passion de la poésie lyrique sous le règne de Néron, à qui le poète Calpurnius Siculus adresse ces vers :

« ne rougis pas d'avoir pincé la corde de la lyre, puisque (...) le farouche Achille luimême, à ce qu'on raconte, a joué de la lyre, alors que le héros fils de Priam [Hector] incendiait mille vaisseaux et que le son grave de la trompette (bucina) couvrait la mélodie des cordes: le héros descendant de Nérée [Achille] fit naître une musique suave des battements de ce pouce fameux ${ }^{40}$ qui lançait la dernière péliade [sa lance] contre l'ennemi » (Eloge de Pison, 169-177 ; trad. J. Amat, CUF).

Bref, lorsqu'un membre des élites envisage de jouer de la lyre, il peut toujours invoquer l'histoire du guerrier Achille afin de rappeler que la pratique des instruments à cordes n'est pas forcément incompatible avec le comportement de l'homme (vir) romain.

\section{Les spécialités musicales chez les musiciens de métier : un partage selon les sexes?}

Dans toutes les cultures, il existe un discours qui réserve certains instruments de musique à l'un des deux sexes. Lorsque l'on aborde cette question à Rome, il faut distinguer soigneusement les musiciens de métier et les dilettantes. Pour les musiciens professionnels dans la société romaine, il n'y a pratiquement aucun partage des sexes puisque l'on connaît à travers les inscriptions des aulètes hommes et femmes (des tibicines et des tibicinae), des joueurs de lyre des deux sexes (fidicines et fidicinae) et des chanteuses (cantrices). Même si les femmes sont largement sous-représentées par rapport aux forts contingents de musiciens masculins (une seule mention dans 
l'épigraphie d'une citharoeda à Rome), la plupart des spécialités musicales se déclinent malgré tout au féminin. C'est même le cas du jeu de l'orgue dans l'Antiquité qui était pratiqué avec un égal bonheur par les hommes et les femmes y compris dans l'arène lors des combats de gladiateurs ${ }^{41}$. Dans certains domaines, ce sont même les femmes qui prédominent en particulier pour tenir le rôle - il est vrai assez peu valorisant - de percussionnistes aux cymbales, au tambourin ou aux crotales (cymbalistriae, tympanistriae ou crotalistriae) et pour assumer la fonction de harpiste. Nous avons la chance de connaître dans l'iconographie d'époque impériale la représentation exceptionnelle d'un "orchestre » qui n'était composé que de femmes grâce à une mosaïque syrienne. Ce pavement de Miriamin daté du IVe $\mathrm{I}^{42}$, est, de ce fait, un document unique dans l'empire : juchées sur une estrade en bois, une organiste, une aulète, une cithariste et deux percussionnistes (une joueuse de crotales et l'autre de bols musicaux) ont été immortalisées en habit des scène sur le pavement d'une salle à manger et c'est à peine si l'unité du groupe est troublée par la présence de deux garçons déguisés en Amours qui actionnent le mécanisme du soufflet de l'orgue pneumatique.

Finalement, dans le monde des professionnels, la seule spécialité pensée comme spécifiquement masculine concerne la trompette qui reste un instrument guerrier. $\mathrm{Si}$ l'on fait exception des Amazones ${ }^{43}$ qui relèvent de la mythologie et de l'inversion du genre, nous ne connaissons dans la littérature qu'une seule femme jouant de la trompe ; encore s'agit-il d'un exemple de l'époque hellénistique (III ${ }^{\mathrm{e}} \mathrm{s}$. av. notre ère) et d'un cas que l'on s'empresse de présenter comme atypique, ce qui valut à cette Aglaïs de Rhodes de passer à la postérité parce qu'elle en avait fait "son métier et son art " (Elien, Histoire variée, I, 26). Mais ce personnage est isolé, et les sources insistent tellement sur sa singularité, que l'on a pu mettre en doute jusqu'à son existence historique ${ }^{44}$.

\section{Des instruments à cordes pour les jeunes filles, mais pas n'importe lesquels...}

Pour l'homme libre, et plus encore pour les femmes, l'éducation musicale contourne et réprouve l'apprentissage de l'aulos (ou tibia) parce que le pincement des anches doubles déformait les traits du visage, selon le souvenir du mythe d'Athéna jetant au loin les auloi sous prétexte qu'ils contribuaient à l'enlaidir (Aristote, Politique, VIII, 14 ; Hygin, Fables, 165). Plusieurs mosaïques romaines ont repris cette image d'Athéna/Minerve se regardant jouer dans une flaque d'eau. La déesse découvre que l'exercice de cet instrument à vent rend ses traits méconnaissables; elle l'abandonne en s'écriant : « loin d'ici tibia, tu ne vaux pas la peine que je te garde! (Ovide, L'Art d'Aimer, III, 47) et il sera récupéré par le satyre Marsyas qui s'en ira braver Apollon et sa lyre. Le souvenir de cette mésaventure est cultivé à l'époque impériale : le jeu de l'aulos déforme le visage et prive l'individu de la parole. De fait, jamais un texte ne nous parle d'un jeune homme ou, pire encore, d'une jeune fille de bonne famille désirant apprendre à souffler dans les auloi. Les femmes aulètes ne sont que des musiciennes professionnelles que leur condition et leur spécialisation mettent à l'abri de telles considérations morales et esthétiques. Là encore, certains auteurs ne se privent pas de glisser quelques allusions grivoises à leur propos sous prétexte qu'elles prennent plaisir à introduire l'anche dans leur bouche. Le poète Martial (Epigrammes, XIV, 63) s'amuse à décrire l'habileté d'une tibicina capable d'emboucher tantôt un, tantôt deux tuyaux à la foi. L'amalgame entre la 
professionnelle de la musique et la courtisane n'est jamais très loin ${ }^{45}$, même si l'on a souvent abusé de ce raccourci.

Dans ses traités sur L'art d'aimer et sur les Remèdes à l'Amour rédigés à l'époque augustéenne, Ovide affirme que la pratique musicale est un aspect valorisant de la féminité ; la femme sans talents est celle « qui n'a pas de voix ", qui « ne sait pas bouger la main » et qui «n'a pas appris à toucher les cordes d'une lyre ». Les scènes dites de leçon de musique peintes sur les murs de Pompéi montrent toujours une jeune fille apprenant le jeu de la lyre ou de la cithara auprès d'un maître selon un motif hérité de la peinture hellénistique. H.I. Marrou a bien expliqué que, sur les sarcophages romains, l'idéal culturel pour les couples des élites prenait la forme de représentations bien codifiées: l'homme est montré avec le volumen, le rouleau, symbole de la culture littéraire, placé dans la main droite ; alors que la femme - et elle seule - tient dans ses bras une lyre ou une cithara, plus rarement un luth à l'époque des Sévères (FIG. 3). Le seul instrument à cordes qui soit exclu de cet univers domestique est la harpe : « je ne veux pas de ces psaltérions et de ces spadix, qu'on devrait même interdire aux jeunes filles honnêtes (virginae probae) » tonne Quintilien (Institution oratoire, I, 10, 31). On peut d'ailleurs constater l'extrême rareté des représentations de harpes dans l'iconographie romaine ${ }^{46}$ alors que les instruments à cordes y sont innombrables. Pourquoi rejeter la harpe qui est pourtant associée dans notre imaginaire au monde féminin depuis l'époque moderne ? Parce qu'elle a dans l'Antiquité une connotation orientale très forte (c'est l'instrument de la Mésopotamie, de la Syrie et de l'Egypte) et qu'à ce titre, elle véhicule les tares de l'Orient : la mollitia et la luxuria, autrement dit les plaisirs lascifs. Voilà qui nous ramène à Clodius...

On pourra constater qu'il n'y a pas adéquation exacte entre la division des instruments de musique établie par Aristide Quintilien et leur adoption par l'un des deux sexes car si la lyre est dépeinte par le théoricien comme relevant du masculin par rapport à la tessiture de la harpe, elle n'en est pas moins un instrument à cordes utilisé par les deux sexes, et même par les femmes de façon très majoritaire. C'est une chose de diviser les instruments de musique selon leur tessiture, c'en est une autre de les répartir selon leur «caractère » supposé et en fonction de la connotation qu'on leur prête. En tout cas, c'est bien à partir de l'Antiquité qu'est instauré ce lien étroit, appelé à durer au cours de l'histoire, entre les jeunes filles de bonne famille et le jeu des instruments à cordes. A n'en pas douter, ces recommandations adressées aux femmes à l'époque de Mozart n'auraient pas été désavouées par Ovide :

\section{BIBLIOGRAPHIE}

« toute jeune fille raffinée, qu'elle ait ou non du talent, doit apprendre à jouer du piano ou à chanter; premièrement c'est la mode; deuxièmement c'est la manière la plus commode de se produire élégamment en société ${ }^{47}$.

ADAMS James Noel, 1982, The Latin Sexual Vocabulary, Londres, Duckworth. 
BALTY Janine, 1977, Mosaïques antiques de Syrie, Bruxelles, Musées Royaux d'Art et d'Histoire.

BARATTE François, 1974, « Un sarcophage d'Achille inédit », Mélanges de l'Ecole Française de Rome, 86, p. 809-810.

BARATTE François et METZGER Catherine, 1985, Catalogue des sarcophages en pierre d'époque romaine et paléochrétienne, Paris, RMN.

BARKER Andrew, 1989, Greek Musical Writings, II. Harmonic and Acoustic Theory, Cambridge University Press.

BÉLIS Annie, 1984, «Un nouveau document musical », Bulletin de Correspondance Hellénique, 108, p. 99-109.

_-, 1986, « L'aulos phrygien », Revue Archéologique, 1986, p. 25-27.

_-, 1996, Les musiciens dans l'Antiquité, Paris, Hachette.

BRIL Jacques, 1980, A cordes et à cri. Origines et symbolisme des instruments de musique, Paris, Clancier /Guénaud.

CÈBE Jean-Paul, 1990, Varron. Satires Ménippées, vol. 9, Rome, Collection de l'EFR.

CHAILLEY Jacques, 1979, La musique grecque antique, Paris, Les Belles Lettres.

CROISILLE Jean-Michel, 1982, Poésie et art figuré de Néron aux Flaviens, I-II, Bruxelles, coll. Latomus.

CUMONT Franz, 1942, Recherches sur le symbolisme funéraire des Romains, Paris, Geuthner.

DUCHESNE-GUILLEMIN Marcelle et ZAQZUQ Abdul R., 1970, «La mosaïque de Miriamin conservée au Musée de Hama », Annales archéologiques Arabes Syriennes, XX, p. 93-105.

DUPONT Florence, 2000, L'orateur sans visage. Essai sur l'acteur romain et son masque, Paris, PUF.

DUPONT Florence et ELOI Thierry, 1994, Les jeux de Priape. Anthologie d'épigrammes érotiques, Paris, Le Promeneur.

,2001, L'érotisme masculin dans la Rome antique, Paris, Belin.

DUYSINX François, 1999, Aristide Quintilien, La Musique. Bibliothèque de la faculté de Philosophie et Lettres de l'Université de Liège, fasc. CCLXXVI.

HOWARD Walther et AURAS Irmgard, 1957, Musique et sexualité, Paris, PUF.

KEMP-LINDEMANN Dagmar, 1975, Darstellungen des Achilleus in der griechischen und römischen Kunst, Bern-Francfort, Lang.

KOSSATZ-DEISMANN Anneliese, 1981, s.v. « Achilleus » dans Lexicon Iconographicum Mythologiae Classicae (LIMC), I, 1, Zürich-Munich, Artemis Verlag.

LINANT de BELLEFONDS Pascale, 1981, s.v. « Agyrtès » dans Lexicon Iconographicum Mythologiae Classicae (LIMC), I, 1, Zürich-Munich, Artemis Verlag.

_-_ , 1985, Sarcophages attiques de la nécropole de Tyr. Une étude iconographique, Paris, Recherches sur les civilisations.

MATHIESEN Thomas J., 1983, Aristides Quintilianus On music in three Books. Translation with Introduction, Commentary and Annotations, New Haven et Londres, Yale University Press.

MAURIN Jean, 1983, « Labor matronalis : aspects du travail féminin à Rome » dans E. Lévy (dir.), La femme dans les sociétés antiques, Strasbourg, Association pour l'étude de la civilisation romaine, p. 139-155. 
MEHEUST Jean, 1971, Stace. Achilléide, Paris, Collection des Universités de France.

MOREAU Philippe, 1982, Clodiana religio. Un procès politique en 61 av. J.-C., Paris, Les Belles Lettres.

PÉCHÉ Valérie, 2002, « Tibicinae, fidicinae, citharistriae, psaltriae : femmes musiciennes de la comédie romaine ", Revue Belge de Philologie et d'Histoire, 80, p. 133-157.

PERROT Jean, 1965, L'orgue de ses origines hellénistiques à la fin du XIII ${ }^{e}$ s., Paris, Picard.

PERNOT Laurent, 2000, La rhétorique dans l'Antiquité, Paris, Le Livre de Poche.

ROBBINS LANDON, H.C., 1990, 1791. La dernière année de Mozart, Paris, Jean-Claude Lattès.

SCHEID John, 1990, «D'indispensables étrangères. Le rôle religieux des femmes à Rome » dans Histoire des femmes en Occident, I, Paris, Plon, p. 404-437.

TAUZIN Aline, 2001, Figures du féminin dans la société maure (Maurétanie), Paris, Karthala.

VENDRIES Christophe, 1999, Instruments à cordes et musiciens dans l'Empire romain, Paris, l'Harmattan.

_-_ , 2006, « Abstinence sexuelle et infibulation des chanteurs dans la Rome impériale » dans F. Prost et J. Wilgaux (éd.), Penser et représenter le corps dans l'Antiquité, Rennes, Presses Universitaires de Rennes, p. 247-261.

VESTERINEN Marjaana, 2003, « Reading Lucian's Peri orcheseos. Attitudes and Approaches to Pantomime » dans L. Pietila-Castren, M. Vesterinen (éd.), Graptai Poikilai (Papers and Monographs of the Finnish Institute at Athens, 8), Helsinski, p. 43-48.

WEBB Ruth, 2005, « Female entertainers in Late Antiquity » dans Pat Easterling et Edith Hall (éd.), Greek and Roman Actors. Aspects of Ancient Profession, Cambridge University Press, p. 282-303.

WINNINGTON-INGRAM Reginald Pepys, 1963 , Aristides Quintilianus. De Musica, Leipzig, Teubner.

\section{NOTES}

1. Bril, 1980 : 125-133. Je renvoie à l'étude de Tauzin $2001: 53-83$ pour l'exemple de la division des sexes dans la musique telle qu'elle est pensée par l'Islam. Je remercie Agnès Fine de m'avoir signalé cet ouvrage. Il existe bien un livre de Howard et Auraas 1957, qui traite des rapports entre musique et sexualité, mais je n'y ai rien trouvé qui puisse nourrir une réflexion concernant notre sujet.

2. J'utilise ici par commodité l'édition récente de Duysinx 1999 tout en modifiant légèrement en certains endroits la traduction de quelques termes techniques. Il existe aussi une édition commentée par Barker 1989 et une autre de Mathiesen 1983, qui reste l'édition de référence. Pour le texte grec: Winnington-Ingram 1963.

3. Duysinx 1999 : 153, note 5.

4. Chailley 1979: 105-106, explique que le terme "mode " n'existe pas en grec et expose la différence avec les modes de la musique moderne.

5. Il existait quinze tropes ou tons échelonnés de demi ton en demi ton du grave à l'aigu.

6. L'aulos est un instrument à vent à anche double que les Romains nomment tibia.

7. Bélis $1986: 25-27$.

8. L'aulos soliste et l'aulos utilisé pour l'accompagnement des chœurs.

9. La tessiture est l'étendue moyenne des notes que peut couvrir un instrument de musique.

10. Dupont $2000: 75$.

11. Dans cette lettre, le philosophe passe en revue tout ce qui se trouve au rebours de la nature. 
12. Sur le caractère efféminé du pantomime : Vesterinen $2003: 43-48$.

13. Voir Webb : 2005, pour la place des femmes dans le théâtre de l'Antiquité tardive.

14. Sur cet aspect : Vendries 2006.

15. Inscriptiones Graecae Urbis Romae, 1034.

16. Pour l'autre versant (le déguisement des femmes en hommes), je renvoie à Clio, 10/1999: Femmes travesties : un mauvais genre.

17. Scheid $1990: 419-421$.

18. Moreau 1982 : 11-15 pour le récit des évènements et pour l'inventaire des sources; Dupont et Eloi 2001 : 133-135, pour la question du costume.

19. Vendries 1999 : 205-207.

20. Pernot 2000 : 163 explique que l'usage des mots d'origine étrangère chez Cicéron servait à stigmatiser l'adversaire et à jouer sur la fibre nationaliste de son auditoire.

21. Moreau $1982: 14$, note 10 .

22. Dupont et Eloi $2001: 115$.

23. Dupont et Eloi $2001: 135$.

24. Voir les indications de Pausanias (I, 6) : « Homère (..) a eu raison aussi à mon sens de montrer la conquête de Skyros par Achille et de ne pas faire comme ceux qui racontent qu'Achille vécut à Skyros au milieu des jeunes filles ".

25. Méheust 1971: 33, notes 4-5: Le traducteur de Stace suppose une filiation entre les deux événements et l'on serait assez enclin à partager son avis.

26. Linant de Bellefonds $1981: 305$.

27. Linant de Bellefonds 1985 : 73 .

28. Croisille 1978, III, pl. 42.

29. Baratte $1974: 809-810$; Croisille $1978, \mathrm{I}: 145$ et $152-154$

30. Balty $1977: 30$ : la question est posée à propos d'une mosaïque de Palmyre.

31. Voir le grand plat du trésor de KaiserAugst du IV ${ }^{\mathrm{e}}$ s. ap. J.-C ; cf. Kossatz-Deismann $1981: \mathrm{n}^{\circ}$ 102 (détail du médaillon : au son de la trompette, Achille déguisé en femme brandit son bouclier). L'œuvre est signée : elle a été réalisée par un artiste grec.

32. Kossatz-Deismann $1981: 155$.

33. Kemp-Lindemann 1975.

34. Maurin 1983 : 146, sur le thème de la matrone filant la laine. Le filage, au même titre que la guerre pour les hommes, est placé sous les auspices de Minerve.

35. Kossatz-Deismann 1981 : 55-68 et Linant de Bellefonds 1981 et 1985 : 71-73 qui fait le point sur la question.

36. Baratte et Metzger 1985 : 255 (Louvre, MA 2120)

37. Voir le commentaire de Cèbe $1990: 1540$; nervia désigne à la fois les cordes et le sexe d'Achille et tractare ( caresser) a aussi un sens érotique.

38. Dupont et Eloi $1994: n^{\circ}$ 68. Les Priapea sont des recueils d'épigrammes anonymes composées vers le $\mathrm{I}^{\mathrm{er}}$ siècle. Pour les rapports entre le langage musical et la sexualité dans la langue latine : cf. Adams, $1982: 25$

39. Cumont $1942: 22$

40. Dans la technique de jeu des instruments à cordes à Rome, le pouce joue un rôle essentiel d'après les témoignages écrits : Vendries 1999 : 181-182.

41. Sur la place des femmes organistes dans l'Antiquité romaine : cf. Perrot $1965: 267$.

42. Duchesne-Guillemin et Zaqzuq 1970 ; Balty 1977 : 94.

43. Bélis, 1984. L'auteur étudie un objet spécifiquement féminin (l'épinètron utilisé pour carder la laine) conservé au Musée d'Eleusis qui présente un décor peint avec des Amazones ; l'une d'entre elles est montrée soufflant dans une trompette accompagnée de signes de notation musicale censés évoquer la sonnerie militaire.

44. Bélis 1999 : 60. 
45. Péché 2002 : 134-135.

46. Vendries 1999 : 105-108.

47. Robbins-Landon $1990: 27$.

\section{RÉSUMÉS}

Dans l'Antiquité romaine, la musique participe à sa façon à la construction du genre en introduisant une division sexuée des notes et des instruments de musique dans la théorie musicale et un partage des sexes lors de la pratique musicale. La question du costume du musicien et du travestissement offre aussi une autre occasion d'explorer le thème du genre. Êtres ambivalents par excellence, le musicien et le danseur se situent parfois en marge du monde masculin à cause de leur allure, de leur habit et de la nature même de leur métier. L'histoire emblématique d'Achille caché dans le gynécée à Skyros ou celle, réelle, du noble Clodius travesti en femme utilisant tous deux les apparences de la musicienne en sont les manifestations les plus abouties qui servent d'exempla pour les Anciens dans la définition des frontières entre féminité et virilité.

In Roman society, music can be interpreted in gendered terms. In music one finds a sexual division of notes, musical instruments, musical theory and musical practice.The musician's clothing and transvesticism can also be studied through the lens of gender. The musician and the dancer are ambivalent by nature because of their appearance, and are often positioned on the fringe of masculinity. In the emblematic story of Achilles, hiding in the gynaeceum of Skyros, or the true story of Claudius in Rome disguised as a woman, both men use the appearance of a woman musician. For the Romans, their stories are considered exempla, defining the borders between femininity and virility.

\section{INDEX}

Keywords : Achilles, Claudius, disguise, feminity, music, Roman Antiquity, virility

Index chronologique : Antiquité romaine

Mots-clés : Achille, Clodius, féminité, gynécée, musique, déguisement 